\title{
Rapid Recognition System of Circuit Breaker Status Based on Wavelet Packet Decomposition and SVM
}

\author{
Jin $\operatorname{Tan}^{1}$, Yao Wei ${ }^{2, b}$ and Mengyuan $\mathrm{He}^{2, \mathrm{c}}$ \\ ${ }^{1}$ Guangdong Electric Power Research Institute, Guangzhou, Guangdong 510600, China \\ ${ }^{2}$ North China Electric Power University, Baoding, Hebei 071003, China \\ bxunxi1527@163.com, 'priangelina@163.com
}

Keywords: Circuit breaker, Wavelet packet decomposition, SVM, Rapid recognition, Fault diagnosis.

Abstract. For circuit breaker failure state is difficult to identify quickly, this paper put forward a rapid recognition method of circuit breaker status based on wavelet packet decomposition and Support Vector Machine(SVM). After db3 wavelet packet decomposition, the extracted circuit breaker vibration signal has a two-stage classification in SVM and the circuit breaker status will be quickly identified. The experimental results show that rapid recognition system of circuit breaker status based on wavelet packet decomposition and SVM can improve the speed of the circuit breaker fault diagnosis effectively without reducing the diagnosis accuracy.

\section{Introduction}

Survey found that $80 \%$ of circuit breaker failure is caused by the poor mechanical properties. At present, the performance of circuit breaker mechanical can be checked only during the regular equipment maintenance, reliability will be affected by frequent removal of circuit breaker. In this paper, a rapid recognition method of circuit breaker status based on wavelet packet decomposition and SVM is proposed, rapid classification on circuit breaker status can improve the speed and accuracy of circuit breaker failure diagnosis. The waste of manpower and resources of the planned maintenance can be reduced, it is of great significance to the safe and stable operation of the power system.

At present, many signal processing method has been applied to fault diagnosis of circuit breaker, such as wavelet analysis ${ }^{[1]}$, the empirical mode decomposition ${ }^{[2]}$, singularity analysis ${ }^{[3]}$, etc. In addition, some mature state recognition method also appear in the circuit breaker fault diagnosis research. There are mainly expert system ${ }^{[4]}$, artificial neural network ${ }^{[5]}$ and other methods.

Most of these traditional algorithm adopt some kind of algorithm which are derived from vibration signals to extract the feature parameters, and then use some sort of pattern recognition methods to classify the fault circuit breaker. Their deficiencies and defects are also exposed in actual application. For example, expert system requires a lot of experience, different rules has a great influence on the final result. While the neural network training samples are difficult to get. There are some reasons, the existing theory of state recognition method is based on statistics, and the gradual theory whose sample size tends to infinity is researched in traditional statistical. These problems cannot be fundamentally solved. In practical engineering, the sample size is usually limited, this limits the application of these methods.

Support Vector Machine (SVM), which is developed based on statistical learning theory, is a kind of special classification algorithm specialized in solving some sorting algorithms whose sample size are small. It solves the defects of the traditional classification method and has a strong theoretical basis. Wavelet packet decomposition and the SVM technology are combined in this paper, and a circuit breaker status identification system are designed. This system can overcome the defect of traditional method, and realize the recognition of the relatively rapid breaker online status. 


\section{Workflow for identify system of circuit breaker status}

First of all, the characteristics of circuit breaker vibration signal are extracted , and decomposed by db3 wavelet packet on three floors. Then the results are input into support vector machine (SVM) to have state classification. Therefore, circuit breaker working condition can be identified. Rapid recognition system workflow of circuit breaker status is shown in Fig. 1.

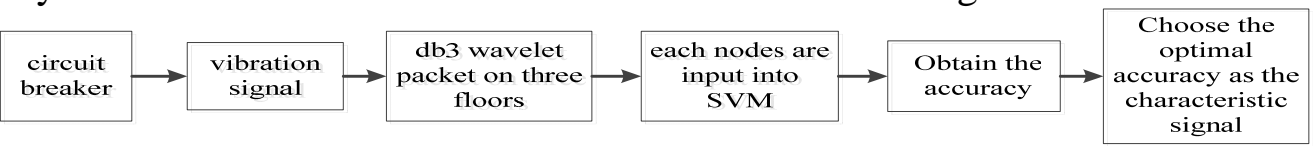

Fig. 1 Rapid recognition system workflow of circuit breaker status

\section{Wavelet Packet Transform}

Due to the transience of circuit breaker break-brake time, direct analysis of time domain signal characteristics is very difficult. As a digital signal microscope, wavelet transform has a amplification local characteristic function which is suitable to find small bursts of abnormal vibration signals during the circuit breaker operation. Compared with the ordinary wavelet transform, wavelet packet not only focuses on approximation signals of signal decomposition, but can also reflect the detail signal distribution in the original signal more intuitive, and keep high resolution at all frequencies.

Wavelet packet transform on signal $W(t)$, definitions are as follows ${ }^{[6]}$.

$\left\{\begin{array}{l}W_{2 n}(t)=\sqrt{2} \sum h_{k} W_{n}(2 t-k) \\ W_{2 n+1}(t)=\sqrt{2} \sum g_{k} W_{n}(2 t-k)\end{array}\right.$

Type: $h$, g are high and low pass filter group respectively.

The breakdown structure is shown in Fig. 2. Within the scope of the band, all subband are divided into two parts of each layer, and spread to the next layer, so as to constitute a binary tree structure. Each layer covers all the signal frequency, but there are different resolution in each layer. More decomposition layers, higher frequency resolution, accordingly, lower temporal resolution ${ }^{[7]}$.

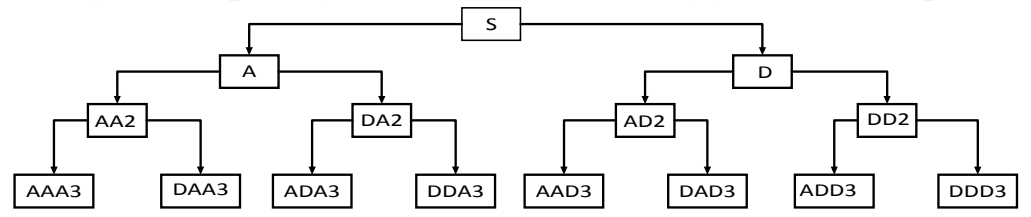

Fig. 2 Wavelet Packet Transform

\section{Support Vector Machine}

High voltage circuit breaker has little action in the operation, the acquisition of vibration signal sample is difficult. Support Vector Machine (SVM) can achieve very good effect of sample classification under the condition of small sample size.

The classification performance of SVM in the fault diagnosis of small sample is superior to other traditional classifier. As is shown in Fig. 3, G is put as an input of the SVM, "a pair of other" model is used for circuit breaker fault diagnosis. This model does not exist classified blind area, for $\mathrm{K}$ classification problems, it needs to have $\mathrm{K}-1$ child classifiers. That is to say, in the simulation experiments, the normal state, the pedestal looseness fault and other fault, a total of three states have a first level classification by SVM1. In the normal state, SVM1 output - 1, and recognition process is over. In the Fault state, output SVM1 + 1. And then the second level classification was adopted by SVM2. In the pedestal looseness fault, SVM2 output - 1, and recognition process is over. In other fault state, output SVM2 + 1, identification process is end. 


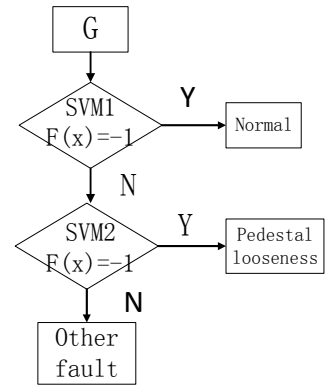

Fig. 3 classification principle of SVM

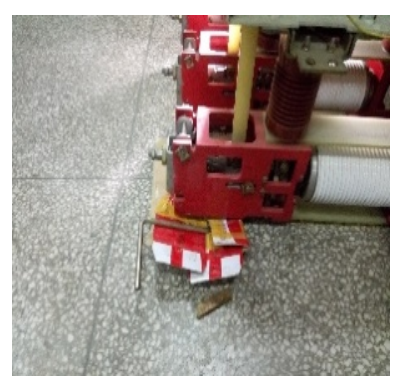

Fig. 4 the pedestal looseness fault

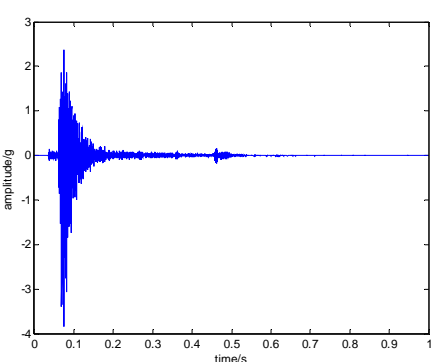

Fig. 5 The original vibration signal

\section{Experimental Analysis}

Signal extraction and processing. ZN65 type vacuum circuit breaker is used as the experimental object. The current sensor on the coil is used as the trigger of acquisition signal in the break-brake experiments. The fault of experimental simulation is the pedestal looseness fault, it is realized by padding circuit breaker one foot, as shown in Fig. 4. There are 19 times break-brake of control circuit breaker in the normal condition and fault condition. Among them, 13 times are in the normal break-brake condition, 6 times are in the brake failure condition. The original vibration signal of the circuit breaker is composed of 20000 points, as is shown in Fig. 5.

After Hilbert transform, the collected vibration signals are descomposed by db3 three layer wavelet packet according to the method of wavelet packet decomposition. Then, eight nodes are get, each node is composed of 2500 points, as shown in Fig. 6, 7.

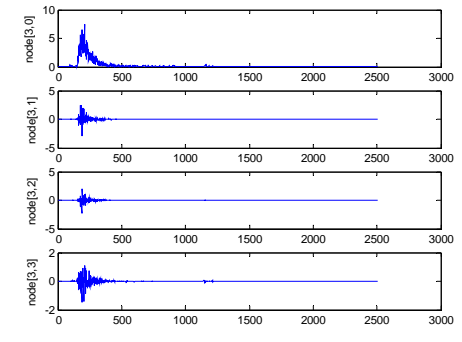

Fig. 6 the first four nodes after wavelet packet decomposition

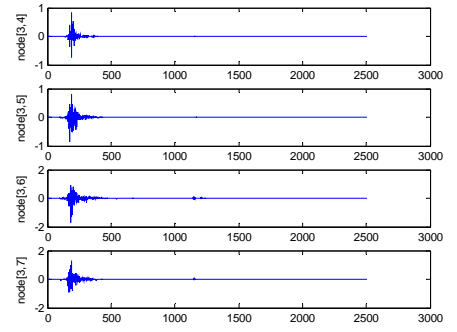

Fig. 7 the last four nodes after wavelet packet decomposition

State recognition results. The node characteristics before and after decomposed are input into support vector machine (SVM) to use for state recognition, and get the recognition accuracy. The results are shown in table 1 . After decomposition, nodes $[3,0]$ to $[3,7]$, the eight nodes for normal circuit breaker status identification accuracy can reach 100\%. And for the state of the circuit breaker pedestal looseness fault identification, $[3,0]$ node identification accuracy can only reach 33.33\%, [3, 7] node can only reach $16.67 \%$, and the other six nodes can't identify the fault. Before the decomposition, nodes $[0,0]$ can identify the normal circuit breaker state at $100 \%$, but it could not identify pedestal looseness fault of the circuit breaker. In conclusion, overall recognition accuracy of $[3,0]$ is higher than the other 7 nodes and the signal characteristics which are decomposed before.

Table 1 Vibration signal wavelet packet node distribution accuracy

\begin{tabular}{|c|c|c|c|c|c|c|c|c|c|}
\hline node & {$[0,0]$} & {$[3,0]$} & {$[3,1]$} & {$[3,2]$} & {$[3,3]$} & {$[3,4]$} & {$[3,5]$} & {$[3,6]$} & {$[3,7]$} \\
\hline normal & $100 \%$ & $100 \%$ & $100 \%$ & $100 \%$ & $100 \%$ & $100 \%$ & $100 \%$ & $100 \%$ & $100 \%$ \\
\hline $\begin{array}{l}\text { pedestal } \\
\text { looseness }\end{array}$ & $0 \%$ & $33.33 \%$ & $0 \%$ & $0 \%$ & $0 \%$ & $0 \%$ & $0 \%$ & $16.67 \%$ & $0 \%$ \\
\hline total & $\begin{array}{c}68.4211 \\
\% \\
\end{array}$ & $\begin{array}{c}78.9474 \\
\% \\
\end{array}$ & $\begin{array}{c}68.4211 \\
\% \\
\end{array}$ & $\begin{array}{c}68.4211 \\
\% \\
\end{array}$ & $\begin{array}{c}68.4211 \\
\% \\
\end{array}$ & $\begin{array}{c}68.4211 \\
\% \\
\end{array}$ & $\begin{array}{c}68.4211 \\
\% \\
\end{array}$ & $\begin{array}{c}73.6842 \\
\% \\
\end{array}$ & $\begin{array}{c}68.4211 \\
\% \\
\end{array}$ \\
\hline
\end{tabular}

At the same time, in the experiments, the operation time of inputing the 2500 points characteristic quantity of the $[3,0]$ into the SVM is $0.026446 \mathrm{~s}$, compared to the operation time of 20000 points 
characteristic quantity of the vibration signal which is needed $0.101495 \mathrm{~s}$, the recognition speed is improved markedly.

\section{Conclusions}

The experimental results show that the change of the vibration signal of circuit breaker operation can reflect circuit breaker operation state. This paper proposed a rapid recognition system of circuit breaker status based on wavelet packet decomposition and SVM. In this system, one can only use the characteristics of $[3,0]$ node to identify the circuit breaker status, which reduces the amount of calculation, improve the ability to quickly identify. It also save the resources and improves the efficiency in practical engineering.

The fault type of circuit breaker is complicated and varied. From the experimental results, it can be seen that there remain a problem. The fault state recognition rate is low. There may be two reasons. One is the discrete degree of vibration signal during the action of the circuit breaker, another is the insufficient training sample number, it worth further studying.

\section{Acknowledgements}

This paper is subsidized by Southern Power Grid science-technology program (GDKJ00000031) (Research of mechanical fault diagnosis technology of circuit breaker spring operating mechanism).

\section{References}

[1]Xiao- Yong ZHOU, Yin-zhong YE. Application of Wavelet Analysis to Fault Diagnosis [D]. Shanghai Maritime University, 2001.(In Chinese)

[2]Jian HUANG, Xiaoguang HU, Yunan GONG. Machinery Fault Diagnosis of High Voltage Circuit Breaker Based on Empirical Mode Decomposition [J]. Proceedings of the CSEE, 2011, 31(12):108-113. (In Chinese)

[3]Xiaoguang HU, Jingmin DAI, Yanchao JI,etc. THE FAULT DIAGNOSIS OF HIGH VOLTAGE BREAKERS BASED ON WAVELET SINGULARITY DETECTION [J]. Proceedings of the CSEE, 2001, 21(5):67-70. (In Chinese)

[4]Jian HUANG, Xiao-guang HU, Yu-nan GONG, Fan YANG. Machinery fault diagnosis expert system for high voltage circuit breaker [J]. Electric Machines and Control, 2011, 15(10):43-49. (In Chinese)

[5]Jianyuan XU, Bin ZHANG, Xin LIN, Bin LI, TENG. Application of Energy Spectrum Entropy Vector Method and RBF Neural Networks Optimized by the Particle Swarm in High-voltage Circuit Breaker Mechanical Fault Diagnosis [J]. High Voltage Engineering, 2012, 38(6):1299-1306. (In Chinese)

[6]Zhonghua GUI, Fengqin HAN. Neural Network Based On Wavelet Packet-CHARACTERISTIC ENTROPY FOR FAULT DIAGNOSIS OF DRAFT TUBE [J]. Proceedings of the CSEE, 2005, 25(4):99-102. (In Chinese)

[7]Laijun SUN , Xiaoguang HU, Yanchao JI , Chao LU. Fault Diagnosis for HV Circuit Breakers with Characteristic Entropy of Wavelet Packet [J]. Automation of Electric Power Systems, 2006, 30(14):62-65. (In Chinese) 